University of Wollongong

Research Online

Australian Institute for Innovative Materials -

Papers

Australian Institute for Innovative Materials

$1-1-2015$

Yolk-shell silicon-mesoporous carbon anode with compact solid electrolyte interphase film for superior lithium-ion batteries

Jianping Yang

University of Wollongong

Yunxiao Wang

University of Wollongong, yunxiao@uow.edu.au

Shulei Chou

University of Wollongong, shulei@uow.edu.au

Renyuan Zhang

Fudan University

Yanfei Xu

University of Wollongong, yx867@uowmail.edu.au

See next page for additional authors

Follow this and additional works at: https://ro.uow.edu.au/aiimpapers

Part of the Engineering Commons, and the Physical Sciences and Mathematics Commons

Research Online is the open access institutional repository for the University of Wollongong. For further information contact the UOW Library: research-pubs@uow.edu.au 


\title{
Yolk-shell silicon-mesoporous carbon anode with compact solid electrolyte interphase film for superior lithium-ion batteries
}

\begin{abstract}
Silicon as an electrode suffers from short cycling life, as well as unsatisfactory rate-capability caused by the large volume expansion ( 400\%) and the consequent structural degradation during lithiation/ delithiation processes. Here, we have engineered unique void-containing mesoporous carbonencapsulated commercial silicon nanoparticles (NPs) in yolk-shell structures. In this design, the silicon NPs yolk are wrapped into open and accessible mesoporous carbon shells, the void space between yolk and shell provides enough room for Si expansion, meanwhile, the porosity of carbon shell enables fast transport of $\mathrm{Li}+$ ions between electrolyte and silicon. Our ex-situ characterization clearly reveals for the first time that a favorable homogeneous and compact solid electrolyte interphase (SEI) film is formed along the mesoporous carbon shells. As a result, such yolk-shell Si@mesoporous-carbon nanoparticles with a large void exhibits long cycling stability (78.6\% capacity retention as long as 400 cycles), and superior rate-capability ( $62.3 \%$ capacity retention at a very high current density of $8.4 \mathrm{Ag}-1)$.
\end{abstract}

\section{Keywords}

interphase, film, superior, lithium, ion, batteries, electrolyte, compact, solid, anode, carbon, mesoporous, yolk, silicon, shell

\section{Disciplines}

Engineering | Physical Sciences and Mathematics

\section{Publication Details}

Yang, J., Wang, Y., Chou, S., Zhang, R., Xu, Y., Fan, J., Zhang, W., Liu, H. Kun., Zhao, D. \& Dou, S. Xue. (2015). Yolk-shell silicon-mesoporous carbon anode with compact solid electrolyte interphase film for superior lithium-ion batteries. Nano Energy, 18 133-142.

\section{Authors}

Jianping Yang, Yunxiao Wang, Shulei Chou, Renyuan Zhang, Yanfei Xu, Jianwei Fan, Weixian Zhang, HuaKun Liu, Dongyuan Zhao, and S X. Dou 


\section{Yolk-shell silicon-mesoporous carbon anode with compact solid electrolyte interphase film for superior lithium-ion batteries}

Jianping Yang, ${ }_{+}^{\mathrm{a}, \mathrm{b}, \mathrm{c}}$ Yunxiao Wang,,${ }^{\mathrm{b}, \mathrm{c}}$ Shulei Chou, ${ }^{\mathrm{b}}$ Renyuan Zhang, ${ }^{\mathrm{c}}$

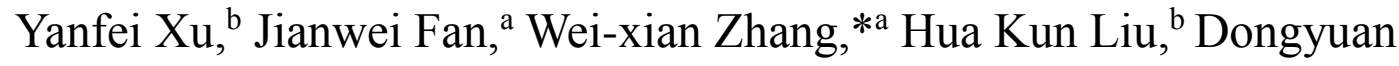
Zhao, ${ }^{* c}$ and Shi Xue Dou*b

a College of Environmental Science and Engineering, State Key Laboratory of Pollution Control and Resources Reuse, Tongji University, Shanghai 200092, P. R. China

b Institute for Superconducting \& Electronic Materials, Australian Institute of Innovative Materials, University of Wollongong, Innovation Campus, Squires Way, North Wollongong, NSW 2500, Australia

c Department of Chemistry, Laboratory of Advanced Materials, Fudan University, Shanghai 200433, P. R. China

$[\dagger]$ These authors contributed equally to this work.

E-mail: zhangwx@tongji.edu.cn (W. Zhang);

dyzhao@fudan.edu.cn (D. Zhao), Tel.: +86-21-5163-0205;

shi_dou@uow.edu.au (S. Dou) 
Abstract: Silicon as an electrode suffers from short cycling life, as well as unsatisfactory rate-capability caused by the large volume expansion $(\sim 400 \%)$ and the consequent structural degradation during lithiation/delithiation processes. Here, we have engineered unique void-containing mesoporous carbon-encapsulated commercial silicon nanoparticles (NPs) in yolk-shell structures. In this design, the silicon NPs yolk are wrapped into open and accessible mesoporous carbon shells, the void space between yolk and shell provides enough room for $\mathrm{Si}$ expansion, meanwhile, the porosity of carbon shell enables fast transport of $\mathrm{Li}^{+}$ions between electrolyte and silicon. Our ex-situ characterization clearly reveals for the first time that a favorable homogeneous and compact solid electrolyte interphase (SEI) film is formed along the mesoporous carbon shells. As a result, such yolk-shell Si@mesoporous-carbon nanoparticles with a large void exhibits long cycling stability (78.6\% capacity retention as long as 400 cycles), and superior rate-capability (62.3\% capacity retention at a very high current density of $8.4 \mathrm{~A} \mathrm{~g} \mathrm{~g}^{-1}$ ).

Keywords: mesoporous carbon, yolk-shell, sol-gel, void space, lithium ion battery 


\section{Introduction}

Developing rechargeable lithium ion batteries (LIBs) with a long cycling life, high energy density, and excellent rate-capability is of critical importance for electric vehicles and renewable energy storage [1-4]. The traditional graphite anode with its limited theoretical capacity of $\sim 372 \mathrm{~mA} \mathrm{~h} \mathrm{~g}^{-1}$, is unable to meet the high energy needs of such applications. Various anode materials and strategies have been explored and proposed for increasing the specific capacity of LIBs [5-7]. Due to its high theoretical capacity $\left(\mathrm{Li}_{4.4} \mathrm{Si}=4200 \mathrm{~mA} \mathrm{~h} \mathrm{~g}{ }^{-1}\right)$ and low discharge potential $\left(\sim 0.5 \mathrm{~V}\right.$ versus $\left.\mathrm{Li} / \mathrm{Li}^{+}\right)$, silicon has been considered as an attractive and promising candidate for LIBs [8]. The practical application of Si-based anodes, however, is still hindered by two critical problems: the relatively low electric conductivity and the huge volume expansion $(\sim$ $400 \%$ upon lithium insertion and extraction [9]. These often cause fracturing, pulverization, and loss of electrical contact, leading to rapid capacity fading and poor cycling performance [10].

It is a common and effective strategy to adopt nanoscale silicon materials with various morphologies, including nanoparticles [11, 12], nanowires [13], nanotubes [14, 15], and hollow spheres $[16,17]$. Compared to bulk silicon, such nanostructured Si is able to accommodate elevated mechanical stress, resulting in prolonged cycling stability. Nanostructured silicon materials, however, still suffer from poor electric conduction [18]. Further optimization is achieved by incorporating nano-silicon materials with various conductive matrixes, such as graphene [19, 20], carbon nanotubes [21], and carbon [22, 23] to form core-shell and yolk-shell nanocomposites [24]. The most promising carbon coating strategy has been explored to promote the electrochemical performance [25-28]. The obvious advantage of carbon shells is intensive improving the overall electrical conductivity of the Si-based anodes. In addition, the introduction of such a carbon shell plays a key role in alleviating the agglomeration of nano-silicon particles [29, 30]. However, the well-established carbon coating methods are based on the chemical vapor deposition, hydrothermal of carbohydrates and polymerization of phenolic resin from sol-gel process, all of them unfortunately result in the compact carbon shells without open and connecting 
mesopore channels for fast transport of $\mathrm{Li}^{+}$ions between the electrolyte and silicon. This could be the reason that Si NPs covered with carbon layer matrix as an anode present unsatisfactory rate-capability [18, 29, 31]. Furthermore, the crucial impact of SEI is usually be neglected and the formation of SEI is hard to control. Importantly, if a stable and compact SEI is constructed, the electrolyte molecules would not penetrate through SEI layer into the active material for further growth, thereby avoiding continual lithium loss and ensuring higher Coulombic efficiency.

Here, aiming at outstanding cycling stability and rate-capability, we report a novel large-void-containing Si@mesoporous carbon yolk-shell structure from commercial silicon NPs for LIB anodes. This unique design has multiple attractive advantages: (i) the special design of the void spaces $(10$ and $50 \mathrm{~nm})$ between the silicon NPs and the mesoporous carbon shells highlights the superiority of the unique yolk-shell structure and allows us to optimize the cycling stability while maintaining a reasonable storage capacity; (ii) the open-ended mesoporous carbon shells with accessible channels are able to facilitate the fast diffusion of $\mathrm{Li}^{+}$ions, and guarantee the full immersion of active materials in the electrolyte, thus contributing to excellent rate capability; (iii) the mesoporous carbon shells are beneficial for the formation of a homogeneous and compact SEI-layer film on the external surface, retaining the internal void space for silicon yolk expansion and rendering superior capacity retention at a high current density. With this design, such elegant mesoporous carbon-encapsulated Si yolk-shell NPs address the challenges of rapid capacity decay and unsatisfied rate capability for Si-anodes, and deliver a high reversible capacity of $\sim 1000 \mathrm{~mA} \mathrm{~h} \mathrm{~g}^{-1}$ with outstanding cyclability during 400 long-deep cycles, as well as superior rate-capability at a very high current density of $8.4 \mathrm{~A} \mathrm{~g}^{-1}$.

\section{Experimental section}

Chemicals: All of the chemicals were analytical grade and used without further purification. Commercial silicon nanoparticles the size ranging from $\sim 60$ to $170 \mathrm{~nm}$ were obtained from Nanostructured and Amorphous Materials, Inc. 2-propanol, ammonia aqueous solution (28 wt \%), tetraethyl orthosilicate (TEOS), 
hexadecyltrimethylammonium bromide (CTAB), $\mathrm{NH}_{4} \mathrm{NO}_{3}, \mathrm{NaOH}, \mathrm{HCl}, \mathrm{HF}$, phenol, and formalin solution (37 wt \%) were purchased from Sinopharm Chemical Reagent Co., Ltd. Deionized water was used in all experiments.

Synthesis of Si@SiO ${ }_{2}$ Core-Shell Nanoparticles: The commercial silicon nanoparticles the size ranging from $\sim 60$ to $170 \mathrm{~nm}$ were first coated with silica layers with different thicknesses according to the Stöber sol-gel method. In a typical synthesis of the $\mathrm{SiO}_{2}$ coating with the thickness of $\sim 50 \mathrm{~nm}, 0.15 \mathrm{~g}$ commercial Si nanoparticles were dispersed in a mixed solution containing deionized water $(70 \mathrm{~mL})$, ethanol $(280 \mathrm{~mL})$, and ammonia (5 mL, $28 \mathrm{wt} \%$ ) under ultrasound for $20 \mathrm{~min}$. Afterwards, $1.0 \mathrm{~mL}$ TEOS was added dropwise every $30 \mathrm{~min}(4.0 \mathrm{~mL}$ in total), and the reaction continued at $25{ }^{\circ} \mathrm{C}$ for $10 \mathrm{~h}$ under continuous mechanical stirring (220 rpm). The core-shell $\mathrm{Si} @ 50 \mathrm{SiO}_{2}$ nanoparticles were obtained after centrifugation and washing with ethanol several times.

For the $\mathrm{SiO}_{2}$ coating with the thickness of $\sim 10 \mathrm{~nm}, 0.1 \mathrm{~g}$ commercial Si nanoparticles were added to $200 \mathrm{~mL}$ 2-propanol, and a homogeneous solution was formed by exposure to ultrasound for $30 \mathrm{~min} .10 \mathrm{~mL}$ ammonia aqueous solution (28 wt \%) and $18 \mathrm{~mL} \mathrm{H}_{2} \mathrm{O}$ were poured into the dispersion and stirred $(600 \mathrm{rpm})$ at $40{ }^{\circ} \mathrm{C}$ for $30 \mathrm{~min}$. Then, 0.1 mL TEOS was injected and reacted for 2 h. The $\mathrm{Si} @ 10 \mathrm{SiO}_{2}$ nanoparticles were collected by centrifugation and washed with ethanol several times.

Synthesis of Si@SiO $@ \mathrm{mSiO}_{2}$ Core-Shell Nanoparticles: The mesoporous silica $\left(m \mathrm{SiO}_{2}\right)$ with thickness of $\sim 15 \mathrm{~nm}$ was deposited on the surface of the $\mathrm{Si} @ \mathrm{SiO}_{2}$ nanoparticles through a well-established surfactant-templating sol-gel route with

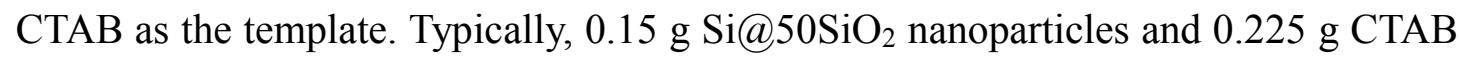
were added into a solution of deionized water $(75 \mathrm{~mL})$, ethanol $(45 \mathrm{~mL})$, and ammonia $(0.825 \mathrm{~mL}, 28 \mathrm{wt} \%)$. The mixture became a homo-geneously dispersed solution after treatment with ultrasound and stirring, for $30 \mathrm{~min}$ each, and then 0.12 $\mathrm{mL}$ TEOS was injected. The reaction was allowed to proceed for $6 \mathrm{~h}$ at $25{ }^{\circ} \mathrm{C}$ under gentle stirring. The products were collected by centrifugation, washed with ethanol, and re-dispersed into $60 \mathrm{~mL} \mathrm{NH} \mathrm{NO}_{3} /$ ethanol $(6 \mathrm{~g} / \mathrm{L})$ solution for removal of the CTAB surfactant. This extraction process proceeded at $60{ }^{\circ} \mathrm{C}$ for $10 \mathrm{~h}$ to yield 
core-shell $\mathrm{Si} @ 50 \mathrm{SiO}_{2} @ m \mathrm{SiO}_{2}$ nanoparticles. The core-shell $\mathrm{Si} @ 10 \mathrm{SiO}_{2} @ m \mathrm{SiO}_{2}$ nanoparticles were synthesized by a method similar to the above process, but with $0.188 \mathrm{~mL}$ TEOS added as the silica source.

Preparation of Phenolic resin Precursors: Low-molecular-weight resol precursors were prepared as the carbon source. Briefly, $8 \mathrm{~g}$ phenol was melted at $45{ }^{\circ} \mathrm{C}$ and mixed with $\mathrm{NaOH}$ aqueous solution (1.36 g, 20 wt \%). This was followed by the dropwise addition of formalin solution (14.2 g, $37 \mathrm{wt} \%)$ under magnetic stirring. Upon further reaction for $1 \mathrm{~h}$ at $70{ }^{\circ} \mathrm{C}$, the solution was cooled to room temperature and adjusted to neutral $\mathrm{pH}(\mathrm{pH} \approx 7)$ with $\mathrm{HCl}(0.6 \mathrm{M})$. The water in the solution was removed by vacuum evaporation at $50{ }^{\circ} \mathrm{C}$.

Synthesis of Si@mC Yolk-Shell Nanoparticles: The mesoporous carbon shell was fabricated via a nanocasting route by using phenolic resin as the carbon source. In a typical procedure, $0.2 \mathrm{~g}$ of $\mathrm{Si} @ 50 \mathrm{SiO}_{2} @ m \mathrm{SiO}_{2}$ nanoparticles was dispersed in $5.0 \mathrm{~mL}$ ethanol, followed by the addition of $1.0 \mathrm{~g}$ of the above-prepared resol. The mixture was stirred at room temperature while open to air to evaporate the ethanol solvent. The dried powder was calcined at $900{ }^{\circ} \mathrm{C}$ for $3 \mathrm{~h}$ under a nitrogen atmosphere to obtain a carbon-covered composite $\left(\mathrm{Si} @ 50 \mathrm{SiO}_{2} @ m \mathrm{SiO}_{2}-\mathrm{C}\right)$. Finally, the mesoporous $\mathrm{SiO}_{2}$ hard template and the $\mathrm{SiO}_{2}$ middle layer were removed to create the mesoporous carbon shell and the void space by etching with $1 \mathrm{wt} \%$ hydrofluoric acid solution. The etching process was conducted overnight at room temperature, followed by a washing with excess deionized water. The resultant yolk-shell Si@50mC nanoparticles were dried at $60{ }^{\circ} \mathrm{C}$ in vacuum for further characterization.

Characterization: The core-shell structures and diameters were further investigated by transmission electron microscopy (TEM, JEOL-2010) operated at an acceleration voltage of $200 \mathrm{kV}$. The samples were suspended in ethanol and dried on a holey carbon film on a $\mathrm{Cu}$ grid for TEM measurements. The morphologies of the samples were observed on a Hitachi S-4800 (Japan) field-emission scanning electron microscope (FESEM). The porosity was measured by nitrogen sorption isotherms at $77 \mathrm{~K}$ with a Micromeritics Tristar 3020 analyzer (USA). Before measurements, the samples were degassed in vacuum at $180{ }^{\circ} \mathrm{C}$ for at least $6 \mathrm{~h}$. The 
Brunauer-Emmett-Teller (BET) method was utilized to calculate the specific surface areas $\left(S_{\mathrm{BET}}\right)$, using adsorption data in a relative pressure $\left(P / P_{0}\right)$ range from 0.04 to 0.2 . The pore volume and pore size distributions were derived from the adsorption branches of isotherms by using the Barrett-Joyner-Halenda (BJH) model. The total pore volume, $V_{\mathrm{t}}$, was estimated from the amount adsorbed at a relative pressure $P / P_{0}$ of 0.995 . The ordering of materials was characterized by small-angle X-ray scattering (SAXS) with a Nanostar U small-angle X-ray scattering system (Bruker, Germany) using $\mathrm{Cu} \mathrm{K \alpha}$ radiation $(\lambda=1.54056 \AA)$. The crystal structure and phase of products were characterized using wide-angle X-ray diffraction (XRD, Bruker D8, Germany) with $\mathrm{Ni}$-filtered $\mathrm{Cu} \mathrm{K} \alpha$ radiation $(40 \mathrm{kV}, 40 \mathrm{~mA})$. The thermal decomposition behavior of the products was monitored by using a Mettler Toledo TGA/SDTA851 analyzer from 50 to $900{ }^{\circ} \mathrm{C}$ in air with a heating rate of $5{ }^{\circ} \mathrm{C} / \mathrm{min}$.

Electrochemical characterizations: The electrochemical measurements were conducted by assembling coin-type half cells in an argon-filled glove box. The electrode slurry was prepared by mixing 60 wt. \% active material (Si NPs, YS Si@10 mC and YS Si@50 mC), 20 wt. \% Super $\mathrm{P}^{\circledR}$, and 20 wt. \% carboxymethyl cellulose $(\mathrm{CMC})$ in a planetary mixer $(\mathrm{KK}-250 \mathrm{~S})$. The electrode films were prepared by pasting the slurry on copper foil using a doctor blade to a thickness of $100 \mu \mathrm{m}$, which was followed by drying in a vacuum oven at $80^{\circ} \mathrm{C}$ overnight. The working electrodes were prepared by punching the electrode film into discs $0.96 \mathrm{~cm}$ in diameter. Lithium foil was employed for both reference and counter electrodes. The electrodes were separated by Celgard separator. The electrolyte was 1.0 M LiPF 6 in 3:4:3 (weight ratio) ethylene carbonate (EC) / dimethyl carbonate (DMC) / diethylene carbonate (DEC), with 5 wt. \% fluoroethylene carbonate (FEC) additive from Novolyte Technologies. The electrochemical performances were tested by a Land Battery Test System with a cut-off voltage range from $0.005 \mathrm{~V}$ to $1.5 \mathrm{~V}$ (vs. $\mathrm{Li} / \mathrm{Li}^{+}$). Cyclic voltammetry and impedance testing were performed using a Biologic VMP-3 electrochemical workstation from $0.005 \mathrm{~V}$ to $1.5 \mathrm{~V}$ at a sweep rate of $0.01 \mathrm{mV} \mathrm{s}^{-1}$.

\section{Results and discussion}


The mesoporous carbon-encapsulated silicon NPs yolk-shell structure can be fabricated through the combination of a sol-gel process and a nanocasting strategy, as schematically shown in Figure 1A. Firstly, the commercial silicon-NPs with the size ranging from $\sim 60$ to $170 \mathrm{~nm}$ (Figure S1A, B) were homogeneously coated with a specially designed sacrificial silica layer via the Stöber sol-gel method using tetraethyl orthosilicate (TEOS) as a precursor in an alkaline alcohol/water system, resulting in the formation of the $\mathrm{Si} @ \mathrm{SiO}_{2}$ core-shell NPs (Figure S1C, D). A thin layer of silicon oxides $\left(\mathrm{SiO}_{x}\right)$ already exists on the surface of the commercial Si NPs, which promote the deposition of silica through physical interaction (Figure S1B). To identify the crucial internal void space to be created after etching away the sacrificial layer and optimize the performance, the thickness of the middle $\mathrm{SiO}_{2}$ sacrificial layer was specially designed to be 10 and $50 \mathrm{~nm}$ by controlling the hydrolysis and condensation of TEOS (Figure 1B, E). Considering the non-uniform size distribution $(\sim 60-170 \mathrm{~nm})$, and their aggregation, the void space of $\sim 10$ and $50 \mathrm{~nm}$ are rationally designed as insufficient and sufficient protection against the volume expansion of $400 \%$ (The theoretical expansion size are evaluated to be $\sim 17.6$ and $49.9 \mathrm{~nm}$ for 60 and $170 \mathrm{~nm}$ Si NPs, respectively). A surfactant-templating sol-gel approach was then used to create a uniform coating of mesoporous silica $\left(m \mathrm{SiO}_{2}\right)$ shells with a thickness of $\sim 15 \mathrm{~nm}$ by using hexadecyltrimethylammonium bromide (CTAB) as a template (Figure 1C, F and Figure S1E, F) [32, 33]. These resultant $\mathrm{Si} @ \mathrm{SiO}_{2} @ \mathrm{mSiO}_{2}$ core-shell NPs have open and worm-like mesopore channels with disordered mesostructures (Figure S2 and S3), which is beneficial for the nanocasting of carbon precursor. This was followed by impregnation of phenolic resin precursor into the mesopore channels through a capillary force and carbonization under nitrogen atmosphere. After selectively leaching out the silica components with the assistance of diluted hydrofluoric acid, the mesoporous carbon-encapsulated Si yolk-shell (YS) structures were obtained and designated as YS Si@xm (where $x$ represents the void space). As revealed by the field-emission scanning electron microscope (FE-SEM) images, the silicon NPs are completely sealed inside the hollow mesoporous carbon $(m \mathrm{C})$ shells (Figure S4). Transmission electron microscope (TEM) observations of 
these YS Si@10mC and YS Si@50mC NPs clearly show the fascinating yolk-shell structures with an internal void space of $\sim 10$ and $\sim 50 \mathrm{~nm}$, originating from the etched middle silica layer. The outer carbon shells have a uniform thickness that is estimated to be about $15 \mathrm{~nm}$, corresponding well to the mesoporous silica layer. Here, the appropriate thickness of the mesoporous carbon was adopted based on the balance between structural stability of shells and superior silicon ratio. Significantly, the carbon shells present highly porous features, accompanied by open, accessible mesopore channels, and disordered mesostructures (Figure 1D, G and Figure S5).

Nitrogen sorption analysis of both YS Si@10mC and YS Si@50mC NPs show type-IV curves with $\mathrm{H} 2$ hysteresis (Figure 2A), demonstrating the presence of mesopores with a uniform size. The pore size distribution peaks strongly center at about $2 \mathrm{~nm}$, measured using the Barrett-Joyner-Halenda (BJH) model (Figure 2B). The Brunauer-Emmett-Teller (BET) surface area and total pore volume are calculated to be as high as $362,582 \mathrm{~m}^{2} \mathrm{~g}^{-1}$ and $0.34,0.51 \mathrm{~cm}^{3} \mathrm{~g}^{-1}$, respectively (Table $\mathrm{S} 1$ ). Additionally, the mesoporous carbon coating was also evaluated by X-ray photoelectron spectroscopy (XPS) and Raman spectroscopy. Compared with the bare commercial Si NPs, a manifest $\mathrm{C}_{1 s}$ peak appears in the XPS survey spectra of YS $\mathrm{Si} @ 10 m \mathrm{C}$ and YS Si@50mC; meanwhile, the intensity of $\mathrm{Si}_{2 s}$ and $\mathrm{Si}_{2 p}$ dramatically decrease (Figure S6). It implies that the Si NPs are embedded in the mesoporous carbon shells, which is in agreement with the TEM images in large domain (Figure S4B, F). Moreover, a significant decrease is observed in the intensity of the characteristic Si Raman bands around $510 \mathrm{~cm}^{-1}$ for the samples YS Si@10mC and YS $\mathrm{Si} @ 50 m \mathrm{C}$, demonstrating the well-sealed structures (Figure 2C). Remarkably, another two broad peaks centered at $\sim 1320$ and $1590 \mathrm{~cm}^{-1}$ are observed, which are assigned to the disorder-induced D band and the graphitic G band, respectively. The strong intensity of $\mathrm{D}$ band indicates the amorphous nature of the mesoporous carbon shells. X-ray diffraction (XRD) patterns of the samples YS Si@10mC and YS Si@50mC display six intense diffraction peaks, similar to commercial silicon NPs with good crystallization. An additional broad peak at about $22.6^{\circ}$ can be observed, which is typical of amorphous carbon in the yolk-shell structured NPs (Figure 2D). This is 
consistent with the Raman results. Furthermore, the mass percentages of silicon in these yolk-shell NPs were determined by thermogravimetric analysis (TGA). The silicon contents are estimated to be $73.4 \%$ and $42.2 \%$ for the samples YS Si@10mC and YS Si@50mC, respectively (Figure S7). Based on the following equation: $Q_{\text {electrode }}=w_{\mathrm{Si}} * Q_{\mathrm{Si}}+w_{m} \mathrm{C}^{*} Q_{m \mathrm{C}}$, where $Q$ and $w$ refer to the theoretical capacity and the mass ratio of silicon and mesoporous carbon, the theoretical capacities of the samples YS Si@10mC and YS Si@50mC are calculated to be 3139 and 1895 mA h g respectively (Figure S8).

The reasonable design of the yolk-shell structures and the optimized free space are manifested by the electrochemical behavior of the electrodes. All the capacities of cells have been normalized based on the weight of active materials in the form of $\mathrm{Si}$ for the pure Si NPs, and yolk-shell Si@mC nanocomposite for the YS Si@10mC and YS Si@50mC. As shown in Figure 3A, the pure commercial Si NPs electrode under-goes a rapid capacity fading, only delivering a reversible capacity of $355 \mathrm{~mA} \mathrm{~h}$ $\mathrm{g}^{-1}$ over 50 cycles. The electrochemical performances of the yolk-shell composites are significantly enhanced. After kinetic enhancement at $140 \mathrm{~mA} \mathrm{~g}^{-1}$ for the initial 5 cycles, YS Si@10mC shows initial reversible capacity of 2014 mA h g ${ }^{-1}$, which accounts for $64.2 \%$ of its theoretical capacity. Its reversible capacity gradually decays to $1440 \mathrm{~mA} \mathrm{~h} \mathrm{~g}^{-1}$ after 50 cycles. In the following cycles, this electrode suffers from severe structural collapse, leading to steep capacity decay. The capacity retention was only remained at $27.7 \%$ after prolonged cycling to 400 cycles. In contrast, YS $\mathrm{Si} @ 50 \mathrm{mC}$ presents a high utilization of $\mathrm{Si}$, achieving a reversible capacity of 1272 $\mathrm{mA} \mathrm{h} \mathrm{g}^{-1}$. Predominant cycling stability is observed, in which the capacity retention is as high as $78.6 \%$ over a long-term 400 cycles. As revealed in the charge and discharge voltage profiles of different cycles (Figure 3B), no obvious capacity decay can be observed in charge profiles at $100^{\text {th }}, 200^{\text {th }}, 300^{\text {th }}$, and $400^{\text {th }}$ cycles. Taking advantages of the sufficient free space $(\sim 50 \mathrm{~nm})$, the designed yolk-shell structure could accommodate the large volume changes in the Si NPs over charge/discharge cycling. As a result, YS Si@50mC is capable of delivering a constant capacity ( $1000 \mathrm{~mA} \mathrm{~h} \mathrm{~g}^{-1}$ ), even over a long-term 400 cycles. Furthermore, when the current 
density increasing from 0.2 to $0.3,0.6,1.5,3$, and $6 \mathrm{C}$ (the current density as high as 8.4 $\mathrm{A} \mathrm{g}^{-1}$ ), the YS Si@50mC NPs present decent specific capacities of 1010, 995, 931, 855,758 , and $620 \mathrm{~mA} \mathrm{~h} \mathrm{~g}^{-1}$, respectively (Figure 3C). It is notable that the sample YS $\mathrm{Si} @ 50 \mathrm{mC}$ completely recovers to the initial reversible capacity $\left(\sim 1000 \mathrm{~mA} \mathrm{~h} \mathrm{~g}^{-1}\right)$ after the heavy-duty cycling, and achieves a stable capacity for the subsequent cycles when the current density recovers to $420 \mathrm{~mA} \mathrm{~g}^{-1}$, further demonstrating its excellent rate capacity retention behavior and cycling sustainability. In contrast, the sample YS Si@10mC shows dramatic capacity fading, indicating the serious destruction of this electrode's structure (Figure S9). The superior electrochemical performance of YS $\mathrm{Si} @ 50 m \mathrm{C}$ is also ascribed to its better SEI film (Figure S10), which exhibits much low charge transfer resistance $\left(R_{c t}\right)$ and leads to fast diffusion of $\mathrm{Li}^{+}$ions through the film. Significantly, it is manifest that the sample YS Si@50mC renders superior rate capacity retention, retaining $62.3 \%$ at a high current of $8.4 \mathrm{~A} \mathrm{~g}^{-1}$, which is favorable compared to other work on Si-anodes reported previously, including hollow Si [16], hollow Si@ppy [34], Si@C sphere [18], Si@C fiber [35], Si@RGO [20], yolk-shell Si@C [22], Si@hydrogel [36], and Si@self-healing-polymer (SHP) [37] (Figure 3D) It is especially noteworthy that YS Si@50m $\mathrm{C}$ is far superior to nonporous carbon encapsulated silicon yolk-shell nanoparticles (YS Si@C) on rate performances, which delivered a capacity retention of $91.3 \%, 80.7 \%, 62.3 \%$ and $64 \%, 52.9 \%, 40.6 \%$ at current density of 2.1, 4.2 and $8.4 \mathrm{~A} \mathrm{~g}^{-1}$, respectively (Figure 3D). The enhancement of rate capacity retention is believed to be ascribed to the advantageous SEI formed with the aid of mesoporous carbon shells.

The SEM image after 100 cycles displays the structural degradation of YS Si@10mC (Figure 4A). The electrode surface shows obvious cracking; and severe agglomeration of the active materials. In contrast, it can be seen from Figure 4B that the YS Si@50mC electrode features a flat and compact surface in large domain, and it maintains its nano-spherical morphology under the same testing conditions. The YS $\mathrm{Si} @ 50 \mathrm{mC}$ electrode well maintains its original structure, which proves that the sufficient void space is able to tolerate volume changes of the inner $\mathrm{Si}$, without causing the shell rupture. Scanning transmission electron microscope (STEM) image 
and energy-dispersive X-ray (EDX) elemental mapping of YS Si@50mC clearly demonstrate that the yolk-shell nanoparticles are preserved and well-contacted with electrolytes during cycling processes (Figure 4C). These structural variations are in agreement with their electrochemical properties and TEM images of YS Si@10mC and YS Si@50mC electrodes after 100 cycles (Figure S11). Similarly, we can draw the same conclusion from the Coulombic efficiency of the electrodes in Figure 4D. The Coulombic efficiency for the Si NPs fluctuates and is less than $95 \%$, indicating significant structural changes in the Si NPs. The Coulombic efficiency of the YS $\mathrm{Si} @ 10 m \mathrm{C}$ is lower than that of YS Si@50mC, and obvious decay appears after 30 cycles, which corresponds to the collapse of the yolk-shell structure and irreversible capacity arising from the formation of updated SEI film. In contrast, the YS Si@50 $m \mathrm{C}$ shows stable and high Coulombic efficiency (>99.1\%) over 100 cycles.

As illustrated in Figure 5A, the buffering space of $10 \mathrm{~nm}$ for the sample YS $\mathrm{Si} @ 10 \mathrm{mC}$ is not enough to accommodate the $\sim 400 \%$ volume expansion of Si NPs within the shell. The mesoporous carbon shells could gradually crack and fracture over subsequent cycling, leading to structural collapse of the designed yolk-shell structure. In comparison, the sufficient free space $(\sim 50 \mathrm{~nm})$ ensures the structure stability and SEI properties of the active materials. The morphology and distribution of SEI of sample YS Si@50mC is characterized at charge state after 3 cycles. A high concentration of lithium layer with the thickness of $\sim 20 \mathrm{~nm}$ is located at the surface of YS Si@50mC NPs in the electrode, which is corresponding to the SEI film (Figure 5B, C). The Li elemental mapping (Figure 5C) demonstrates that a homogeneous and compact SEI layer is observed along the surface of mesoporous carbon surface instead of the silicon particle. Scanning TEM image of single Si@50mC nanoparticle after 3 cycles and the corresponding Li concentration mapped by electron energy loss spectroscopy (EELS) has shown in Figure 5D and 5E. The EELS spectrum from the outer surface of mesoporous carbon shells shows two typical peaks of the Li K-edge. It corresponds to LiF originating from SEI film (Figure 5F), which results from the decomposition of $\mathrm{LiPF}_{6}$ electrolyte during lithiation [38]. It is obvious that no $\mathrm{Li}$ $\mathrm{K}$-edge signal is collected around the lithiated silicon nanoparticle yolk. These results 
further prove that the mesoporous carbon shells are not fully accessible to the electrolyte, probably ascribed to the randomness of the mesopores. This elaborate open-ended carbon shells are favorable to the surface infiltration of electrolyte, the favorable SEI film growes due to the anxo-action of mesopores, in which electrolyte is prone to be soaked in and diminutive SEI film is initially formed around the wall of each pore. As a result, a compact SEI layer is produced along the mesoporous carbon shells. Meanwhile, it is well known that the SEI film does not conduct electrons and is almost impenetrable to electrolyte molecules, the electrolyte, therefore, is almost unable to travel through the SEI layer to the Si yolk surface. Combing with the free space design to accommodate the volume expansion of $\mathrm{Si}$, this synergistic effect guarantee the unique yolk-shell Si@mesoporous carbon composites can successfully tackle the two major problems of silicon anode, including the instability of the SEI and the mechanical degradation caused by the large volume changes.

\section{Conclusions}

In summary, we have rationally designed and successfully fabricated void-containing mesoporous carbon-encapsulated commercial silicon NPs with a yolk-shell structure for stable LIBs. The void space between the Si yolk and the mesoporous carbon shell is reasonably engineered to accommodate the large volume expansion during lithiation and delithiation processes, and optimize the cycling stability. The accessible porosity of the mesoporous carbon shells provides a high electrode-electrolyte contact area, and fast $\mathrm{Li}^{+}$penetration. The yolk-shell structured YS Si@50mC mesoporous carbon composites with an enough void space NPs yield long cycle life ( $1000 \mathrm{~mA} \mathrm{~h} \mathrm{~g}^{-1}$ after 400 cycles $)$ and excellent rate-capability $(62.3 \%$ capacity retained at a high current of $8.4 \mathrm{~A} \mathrm{~g} \mathrm{~g}^{-1}$ ). Furthermore, a homogeneous and compact SEI layer is firstly observed on the surface of mesoporous carbon shells, which could be responsible for the superior rate-capability and stability. This work thus offers a promising design for the structure of other anode materials with long cycling performance and good rate capacity retention.

\section{Acknowledgements}


We acknowledge financial support from State Key Laboratory of Pollution Control and Resource Reuse Foundation (PCRRF14017), the China Postdoctoral Science Foundation (2014M551455 and 2015T80451), the Commonwealth of Australia through the Automotive Australia 2020 Cooperative Research Centre (Auto CRC 2020), and Baosteel-Australia joint research and development centre. The authors thank the Electron Microscopy Centre (EMC) at the University of Wollongong for the electron microscopy characterizations and Dr. T. Silver for critical reading of this manuscript.

\section{Notes and references}

[1] J.M. Tarascon, M. Armand, Nature 414 (2001) 359-367.

[2] K.S. Kang, Y.S. Meng, J. Breger, C.P. Grey, G. Ceder, Science 311 (2006) 977-980.

[3] N.S. Choi, Z.H. Chen, S.A. Freunberger, X.L. Ji, Y.K. Sun, K. Amine, G. Yushin, L.F. Nazar, J. Cho, P.G. Bruce, Angew. Chem. Int. Ed. 51 (2012) 9994-10024.

[4] J.B. Goodenough, K.S. Park, J. Am. Chem. Soc. 135 (2013) 1167-1176.

[5] Y.G. Guo, J.S. Hu, L.J. Wan, Adv. Mater. 20 (2008) 2878-2887.

[6] P.G. Bruce, B. Scrosati, J.M. Tarascon, Angew. Chem. Int. Ed. 47 (2008) 2930-2946.

[7] L.W. Ji, Z. Lin, M. Alcoutlabi, X.W. Zhang, Energy Environ. Sci. 4 (2011) 2682-2699.

[8] R. Teki, M.K. Datta, R. Krishnan, T.C. Parker, T.M. Lu, P.N. Kumta, N. Koratkar, Small 5 (2009) 2236-2242.

[9] H. Wu, Y. Cui, Nano Today 7 (2012) 414-429.

[10] B. Key, M. Morcrette, J.M. Tarascon, C.P. Grey, J. Am. Chem. Soc. 133 (2011) 503-512.

[11] U. Kasavajjula, C.S. Wang, A.J. Appleby, J. Power Sources 163 (2007) 1003-1039.

[12] H. Kim, M. Seo, M.H. Park, J. Cho, Angew. Chem. Int. Ed. 49 (2010) 2146-2149.

[13] M.Y. Ge, J.P. Rong, X. Fang, C.W. Zhou, Nano Lett. 12 (2012) 2318-2323.

[14] W. Wang, P.N. Kumta, ACS Nano 4 (2010) 2233-2241.

[15] M.H. Park, M.G. Kim, J. Joo, K. Kim, J. Kim, S. Ahn, Y. Cui, J. Cho, Nano Lett. 9 (2009) 3844-3847.

[16] X.K. Huang, J. Yang, S. Mao, J.B. Chang, P.B. Hallac, C.R. Fell, B. Metz, J.W. Jiang, P.T. Hurley, J.H. Chen, Adv. Mater. 26 (2014) 4326-4332.

[17] H. Ma, F.Y. Cheng, J. Chen, J.Z. Zhao, C.S. Li, Z.L. Tao, J. Liang, Adv. Mater. 19 (2007) 4067-4070.

[18] W. Li, Y. Tang, W. Kang, Z. Zhang, X. Yang, Y. Zhu, W. Zhang, C.-S. Lee, Small 11(2014) 1345-1351.

[19] X.S. Zhou, Y.X. Yin, L.J. Wan, Y.G. Guo, Adv. Energy Mater. 2 (2012) 1086-1090.

[20] J. Chang, X. Huang, G. Zhou, S. Cui, P.B. Hallac, J. Jiang, P.T. Hurley, J. Chen, Adv. Mater. 26 (2014) 758-764.

[21] L.F. Cui, L.B. Hu, J.W. Choi, Y. Cui, ACS Nano 4 (2010) 3671-3678.

[22] N. Liu, H. Wu, M.T. McDowell, Y. Yao, C.M. Wang, Y. Cui, Nano Lett. 12 (2012) 3315-3321.

[23] S.H. Ng, J.Z. Wang, D. Wexler, K. Konstantinov, Z.P. Guo, H.K. Liu, Angew. Chem. Int. Ed. 45 (2006) 6896-6899.

[24] X.W. Lou, L.A. Archer, Z. Yang, Adv. Mater. 20 (2008) 3987-4019.

[25] J. Liu, S.Z. Qiao, H. Liu, J. Chen, A. Orpe, D. Zhao, G.Q. Lu, Angew. Chem. Int. Ed. 50 (2011) 5947-5951. 
[26] G. He, S. Evers, X. Liang, M. Cuisinier, A. Garsuch, L.F. Nazar, ACS Nano 7 (2013) 10920-10930.

[27] J. Wang, W. Li, F. Wang, Y. Xia, A.M. Asiri, D. Zhao, Nanoscale 6 (2014) 3217-3222.

[28] X. Ma, M. Liu, L. Gan, P.K. Tripathi, Y. Zhao, D. Zhu, Z. Xu, L. Chen, Phys. Chem. Chem. Phys. 16 (2014) 4135-4142.

[29] R. Zhang, Y. Du, D. Li, D. Shen, J. Yang, Z. Guo, H.K. Liu, A.A. Elzatahry, D. Zhao, Adv. Mater. 26 (2014) 6749-6755.

[30] L.W. Su, Y. Jing, Z. Zhou, Nanoscale 3 (2011) 3967-3983.

[31] Y. Ren, A.R. Armstrong, F. Jiao, P.G. Bruce, J. Am. Chem. Soc. 132 (2010) 996-1004.

[32] J. Yang, D. Shen, L. Zhou, W. Li, X. Li, C. Yao, R. Wang, A.M. El-Toni, F. Zhang, D. Zhao, Chem. Mater. 25 (2013) 3030-3037.

[33] J. Yang, D. Shen, L. Zhou, W. Li, J. Fan, A.M. El-Toni, W.-x. Zhang, F. Zhang, D. Zhao, Adv. Healthcare Mater. 3 (2014) 1620-1628.

[34] F.H. Du, B. Li, W. Fu, Y.J. Xiong, K.X. Wang, J.S. Chen, Adv. Mater. 26 (2014) 6145-6150.

[35] T.H. Hwang, Y.M. Lee, B.S. Kong, J.S. Seo, J.W. Choi, Nano Lett. 12 (2012) 802-807.

[36] H. Wu, G.H. Yu, L.J. Pan, N.A. Liu, M.T. McDowell, Z.A. Bao, Y. Cui, Nat. Commun. 4 (2013) 6.

[37] C. Wang, H. Wu, Z. Chen, M.T. McDowell, Y. Cui, Z. Bao, Nat. Chem. 5 (2013) 1042-1048.

[38] H. Ryu, T. Kim, K. Kim, J.-H. Ahn, T. Nam, G. Wang, H.-J. Ahn, J. Power Sources 196 (2011) 5186-5190. 


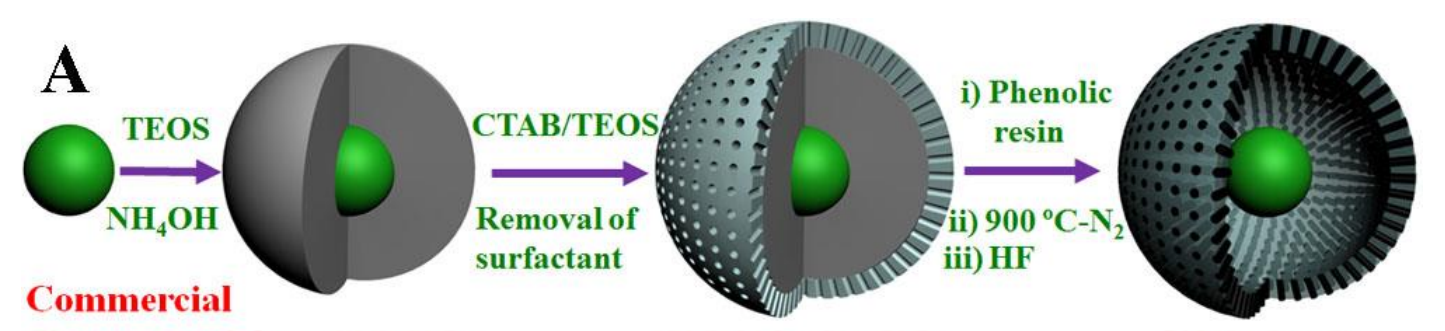

Si nanoparticles $\quad \mathrm{Si} \propto \mathrm{SiO}_{2}$
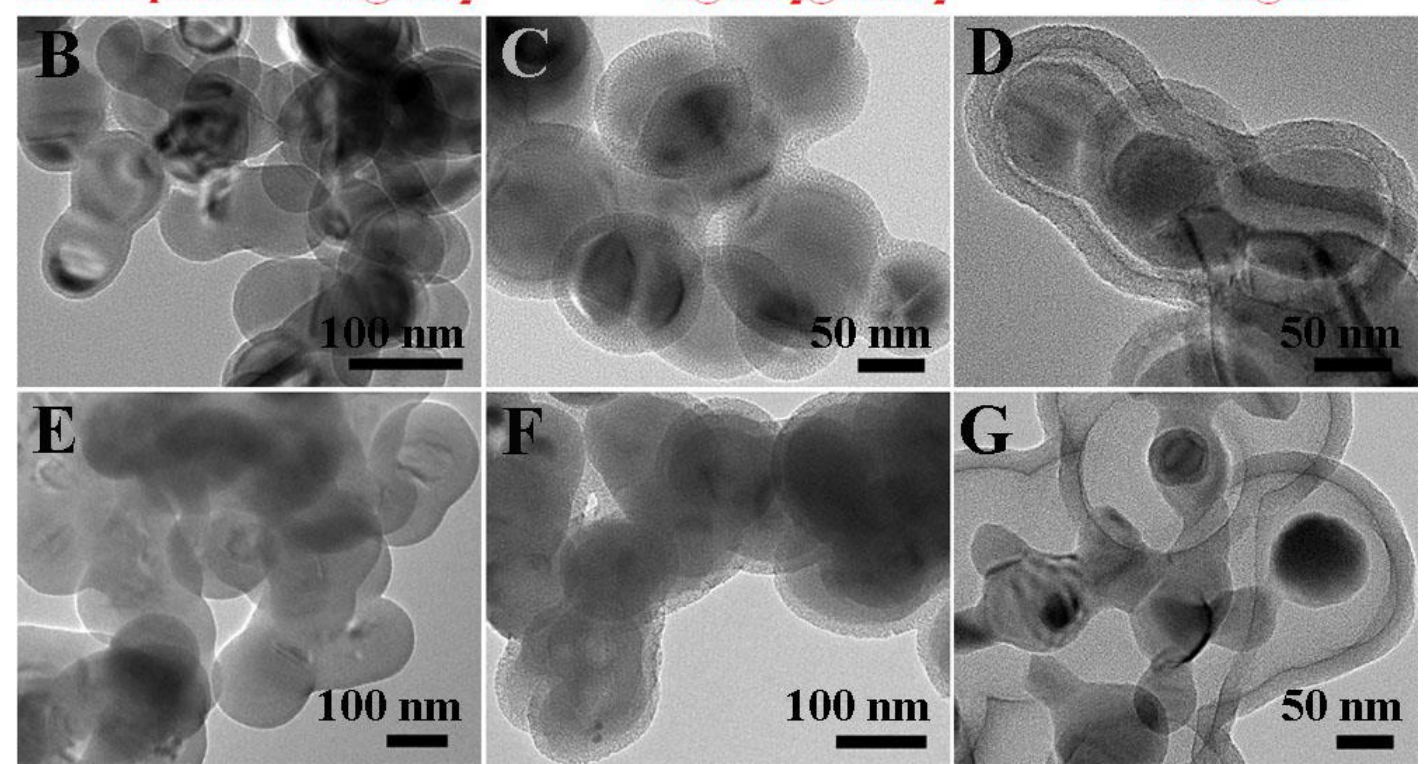

Figure 1 (A) Schematic illustration of the formation process of the mesoporous carbon-encapsulated commercial silicon yolk-shell NPs. (B-G) TEM images of the silica-coated commercial silicon (B) $\mathrm{Si} @ 10 \mathrm{SiO}_{2}$ and (E) $\mathrm{Si} @ 50 \mathrm{SiO}_{2}$ core-shell NPs with the thickness of 10 and $50 \mathrm{~nm}$, respectively; the mesoporous silica coated (C) $\mathrm{Si} @ 10 \mathrm{SiO}_{2} @ m \mathrm{SiO}_{2}$ and $(\mathrm{F}) \mathrm{Si} @ 50 \mathrm{SiO}_{2} @ m \mathrm{SiO}_{2}$ core-shell NPs; the mesoporous carbon-encapsulated commercial silicon (D) YS Si@10mC and (G) YS Si@50mC yolk-shell structure with the void space of 10 and $50 \mathrm{~nm}$, respectively. 

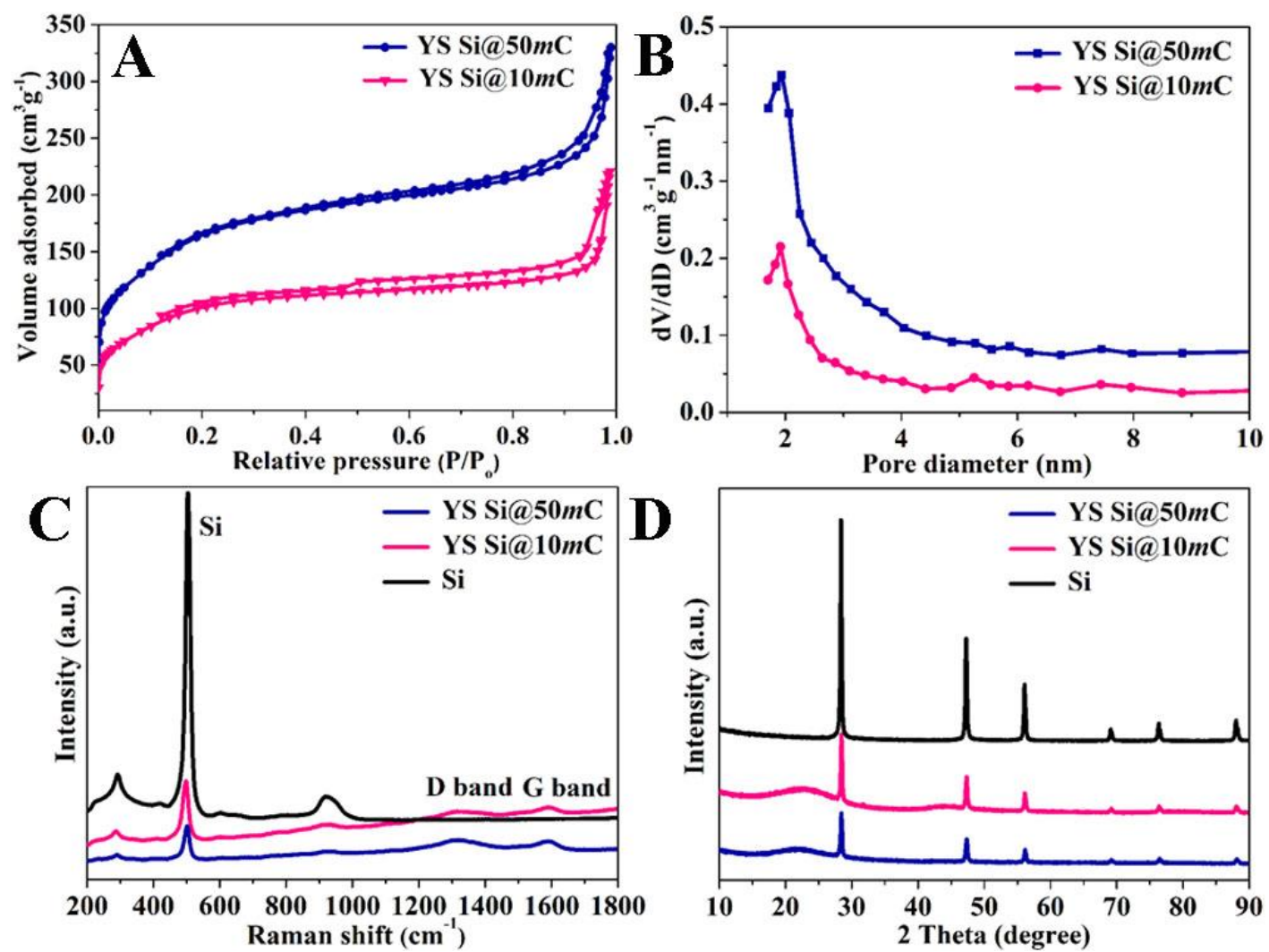

Figure 2 (A) Nitrogen sorption isotherms, (B) pore size distribution curves, (C) Raman spectra, and (D) XRD patterns of the representative samples YS Si@10mC and YS Si@50mC NPs. 

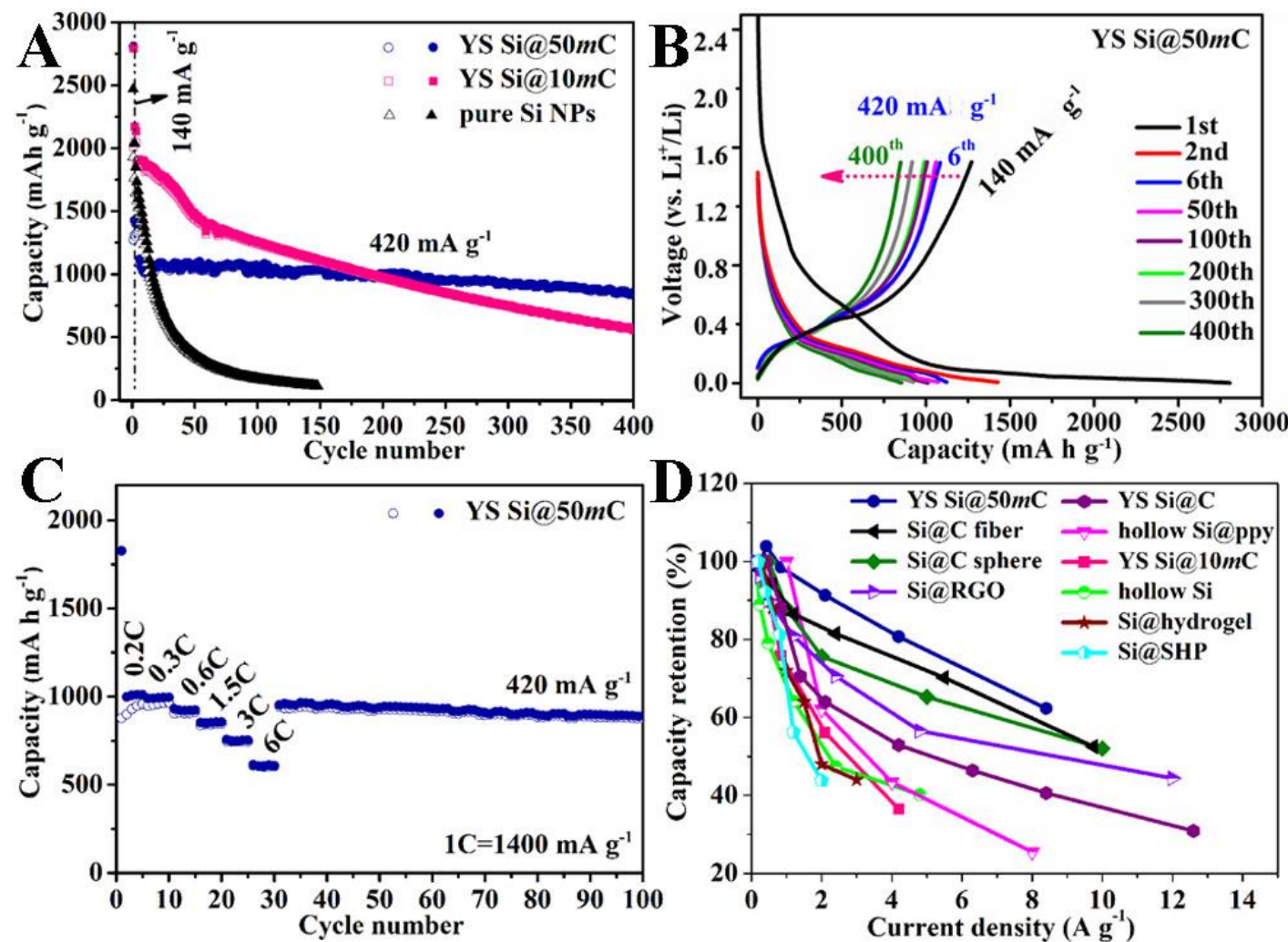

Figure 3 (A) Charge-discharge cycling performance of two represented samples YS Si@50mC, YS Si@10mC, and commercial Si NPs electrodes. (B) Charge-discharge potential profiles of the samples YS Si@50mC at different cycles at a current density of $420 \mathrm{~mA} \mathrm{~g}^{-1}$. (C) Rate performance of YS Si@50mC. (D) Rate capacity retention of YS Si@50mC, YS Si@10mC, Si@C fiber, Si@C sphere, Si@RGO, YS Si@C, hollow Si@ppy, hollow Si, Si@hydrogel, and Si@SHP electrodes cycled at various current densities. 


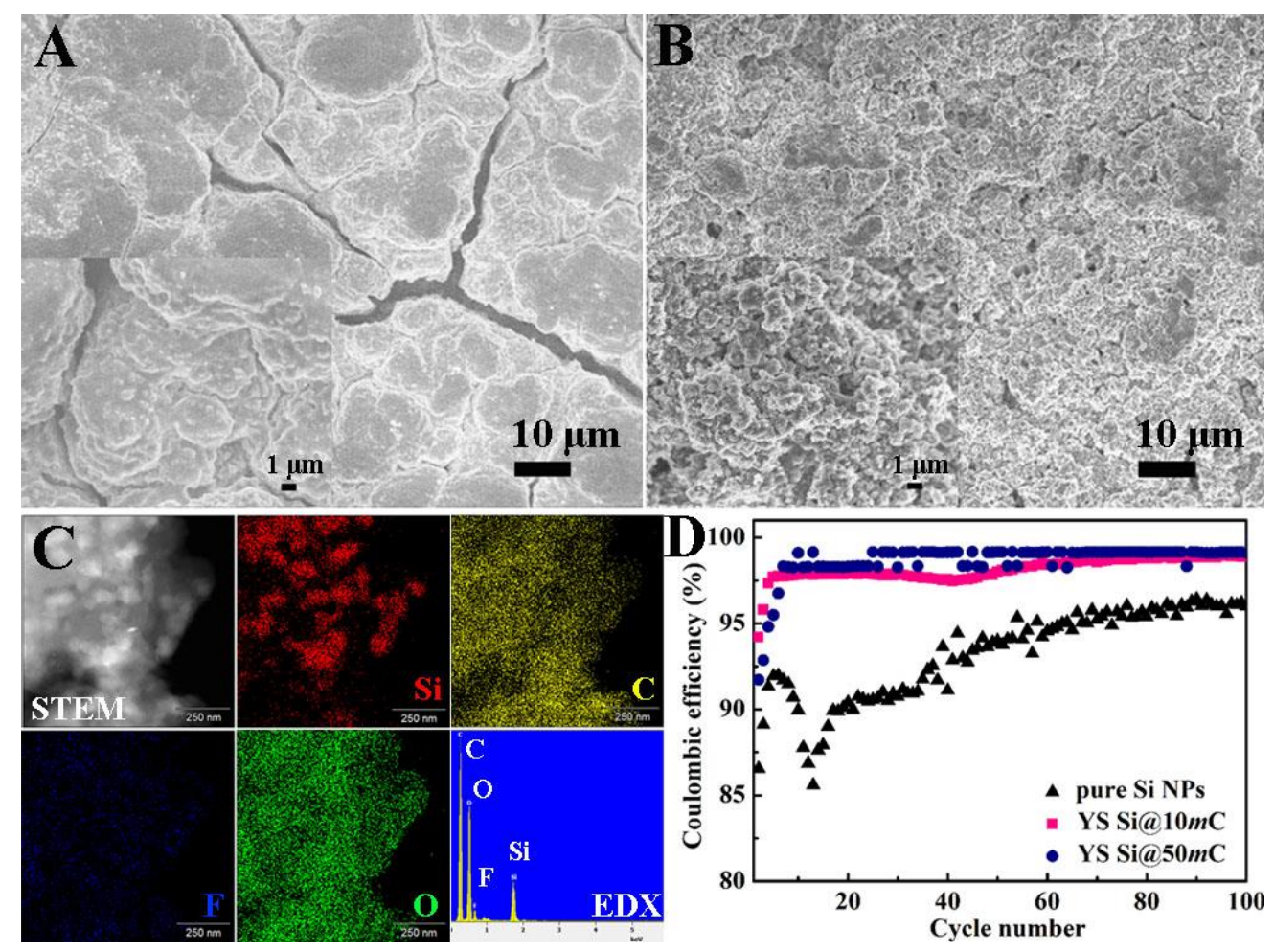

Figure 4 SEM images (A) of YS Si@ $10 m \mathrm{C}$; and (B) YS Si@50mC nanoparticle electrodes; (C) scanning transmission electron microscope (STEM), and energy-dispersive X-ray (EDX) elemental mapping of YS Si@50m C for $\mathrm{Si}, \mathrm{C}, \mathrm{F}$, and $\mathrm{O}$, respectively, and EDX spectrum after 100 cycles at a current density of $420 \mathrm{~mA} \mathrm{~g}^{-1}$. (D) Coulombic efficiency of pure Si NPs, YS Si@10mC, and YS Si@50mC. 


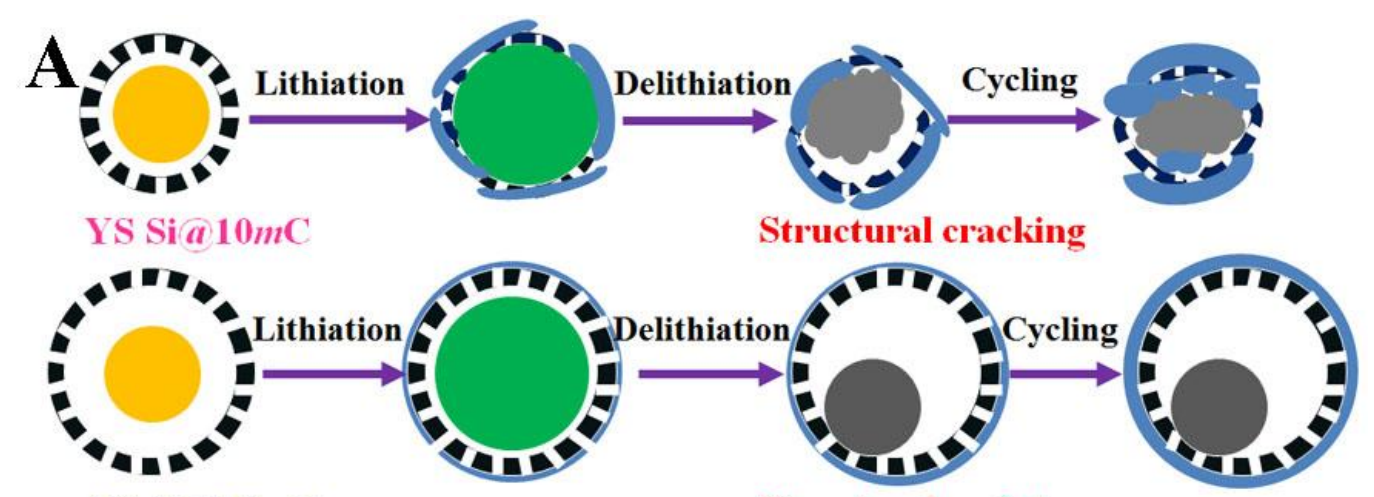

YS Si $\propto 50 m \mathrm{C}$

Structural maintenance
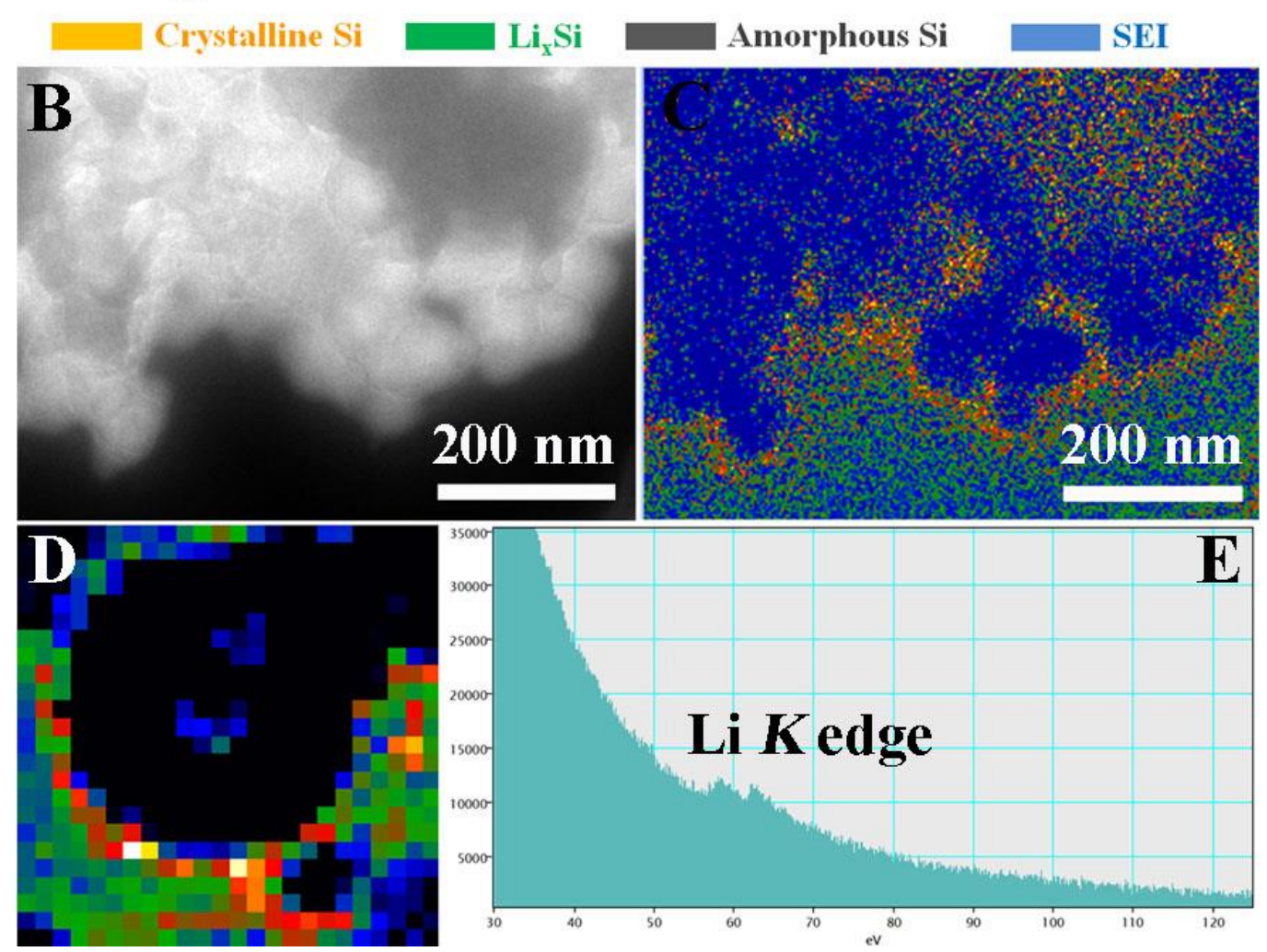

Figure 5 (A) Schematic illustration of lithiation and delithiation processes of YS Si@10mC and YS Si@50mC electrodes. (B) High-angle annular dark field scanning transmission electron microscope (HAADF-STEM) image (scale bar $200 \mathrm{~nm}$ ), and (C) Li elemental mapping (yellow dots) (scale bar $200 \mathrm{~nm}$ ). (D) The scanning TEM (STEM) image of single YS Si@50mC nanoparticle at charge state after 3 cycles, the corresponding (E) Li concentration mapped by EELS (blue color corresponding to low concentration of Li $K$-edge, red color corresponding to high concentration of Li $K$-edge); and (F) EELS spectrum of Li $K$-edge from the surface layer. 